\title{
Impact of Power Control and Lognormal Shadowing on the Mean Transmit Power of Bluetooth Devices
}

\author{
Kun-Wah Yip, Member, IEEE, and Tung-Sang Ng, Fellow, IEEE
}

\begin{abstract}
This letter analyzes Bluetooth's power-control algorithm with a goal to study the mean transmit power required in the presence of lognormal shadowing. The following results are found. 1) A smaller power-control step size yields a lower mean transmit power. 2) When the standard deviations of lognormal shadowing are 3 and $6 \mathrm{~dB}$, respectively, a Bluetooth device needs to consume 11.1 dB and 15.0 dB more in the transmit energy than the minimum one required in the absence of shadowing. 3) The transmit energy consumption varies by around $6 \mathrm{~dB}$ among Bluetooth devices as a result of the $\pm 6 \mathrm{~dB}$ tolerance in the Golden Receive Power Range, which has a nominal size of $20 \mathrm{~dB}$.
\end{abstract}

Index Terms-Bluetooth, power control, shadowing.

\section{INTRODUCTION}

$\mathbf{I}$ T IS POSSIBLE to minimize the transmit power of Bluetooth devices by using feedback power control that is elaborated in the Bluetooth specification [1]. For devices used in homes and offices, shadowing loss due to the movement of people inside buildings has a significant impact on the mean transmit power. Evaluating this impact becomes important as the battery support life of portable Bluetooth equipment can be estimated. However, previous literature on feedback power control, e.g., [2]-[4], has been mainly focused on the capacity increase of cellular CDMA networks and cannot be used to compute the mean transmit power for Bluetooth devices. In this letter, we analyze Bluetooth's power-control algorithm with a goal to study the mean transmit power required in the presence of shadowing. Lognormal shadowing, appropriate for indoor communications [5], is considered.

\section{ANALYSIS}

The power-control algorithm [1] is reviewed as follows. At the receiver, the received signal strength in $\mathrm{dBm}, J_{r}$, is measured and compared against the lower and upper threshold levels in $\mathrm{dBm}$, denoted by $J_{r}^{(\mathrm{LT})}$ and $J_{r}^{(\mathrm{UT})}$, respectively. The lower threshold level specifies the minimum power level that yields an acceptable performance. If $J_{r}$ is lower than $J_{r}^{(\mathrm{LT})}$, a request to increase the transmit power by $\Delta \mathrm{dB}$ is sent from the receiver to the transmitter. If $J_{r}$ is higher than $J_{r}^{(\mathrm{UT})}$, a request to decrease the transmit power by $\Delta \mathrm{dB}$ is issued. The specification [1] states that the allowable step size $\Delta$ ranges from 2 to $8 \mathrm{~dB}$

Manuscript received June 18, 2002. The associate editor coordinating the review of this letter and approving it for publication was Dr. N. Van Stralen. This work was supported by the Hong Kong Research Grants Council and by the University Research Committee of The University of Hong Kong.

The authors are with the Department of Electrical and Electronic Engineering, The University of Hong Kong, Hong Kong (e-mail: kwyip@eee.hku.hk; tsng@eee.hku.hk).

Digital Object Identifier 10.1109/LCOMM.2002.807437 and the Golden Receive Power Range (GRPR), $J_{r}^{(\mathrm{UT})}-J_{r}^{(\mathrm{LT})}$, has a nominal size of $20 \mathrm{~dB}$ with an accuracy of $\pm 6 \mathrm{~dB}$.

We consider the situation that power control is used to compensate for the path loss and shadowing but not for fast fading. In Bluetooth systems, fast fading can be more easily compensated for by frequency hopping. The mean received power $J_{r}$, obtained by averaging the received power measured over a sufficient number of frequency hops, is then used to determine if an increase or decrease of transmit power is required.

In the presence of lognormal shadowing, $J_{r}=$ $J_{t}+10 \log _{10} G$ where $J_{t}$ is the transmit power in $\mathrm{dBm}$ and $G$ is the channel gain modeled by a lognormal random variable. Let $\mu_{\mathrm{dB}}$ and $\sigma_{\mathrm{dB}}$ be the mean and standard deviation, respectively, of $10 \log _{10} G$. Note that $-\mu_{\mathrm{dB}}$ is the mean path loss of the channel and $\sigma_{\mathrm{dB}}$ is a measure of the shadowing depth. In addition,

$$
J_{r}=J_{t}+\mu_{\mathrm{dB}}+\sigma_{\mathrm{dB}} U
$$

where $U$ is a standard normal random variable. The mean channel gain, $\bar{G}$, is given by [6]

$$
\bar{G}=e^{\gamma \mu_{\mathrm{dB}}+\gamma^{2} \sigma_{\mathrm{dB}}^{2} / 2}
$$

where $\gamma=(\ln 10) / 10$ is a scale factor. In the absence of $\log$ normal shadowing, the minimum transmit power in $\mathrm{mW}$ required to achieve an acceptable performance, $W_{t}^{(\mathrm{min})}$, is computed by

$$
W_{t}^{(\min )}=\bar{G}^{-1} 10^{J_{r}{ }^{(\mathrm{LT})} / 10} .
$$

Our task is to compute the mean transmit power in the presence of shadowing, $\overline{W_{t}}$ and compare it to $W_{t}^{(\min )}$. That is, we evaluate

$$
A_{\mathrm{dB}}=10 \log _{10}\left(\frac{\overline{W_{t}}}{W_{t}^{(\min )}}\right) \mathrm{dB} .
$$

Since the amount of transmit power to be increased or decreased is determined by the current level of mean received power, which is also related to the current transmit power level by (1), it is possible to evaluate $A_{\mathrm{dB}}$ by considering a Markov chain. Consider a Markov chain with states numbered from $-M^{\prime}$ to $M$ where $M^{\prime}$ and $M$ are to be determined. Let $J_{t}^{(\min )}$ be the $\mathrm{dBm}$ value of $W_{t}^{(\mathrm{min})}$, given by

$$
J_{t}^{(\min )}=J_{r}^{(\mathrm{LT})}-\mu_{\mathrm{dB}}-\frac{\gamma \sigma_{\mathrm{dB}}^{2}}{2} \mathrm{dBm} .
$$

The state $i$ refers to the case that $J_{t}$ is in the range $J_{t} \in H_{i}$, where $H_{i}=\left[J_{t}^{(\min )}+i \Delta, J_{t}^{(\min )}+(i+1) \Delta\right)$ for $-M^{\prime} \leq$ 
$i \leq M$. Let $F_{n}=\left[J_{r}^{(\mathrm{UT})}+(n-1) \Delta, J_{r}^{(\mathrm{UT})}+n \Delta\right)$ and $G_{n}=\left[J_{r}^{(\mathrm{LT})}-n \Delta, J_{r}^{(\mathrm{LT})}-(n-1) \Delta\right)$ where $n \geq 1$. Assume that the shadowing is slowly varying such that the power-control mechanism can successfully respond to it. If $J_{r} \in G_{n}$, the power-control algorithm drives the transmit power to increase by $n \Delta \mathrm{dB}$ in order that the adjusted $J_{r}$ is within $J_{r}^{(\mathrm{LT})}$ and $J_{r}^{(\mathrm{UT})}$. It follows that the system is transited from the state $i$ to the state $i+n$ unless the transmit power reaches the maximum. In this case, the system reaches the terminating state, i.e., the state $M$. Thus, $M$ can be determined from the maximum output power of a Bluetooth device. Similar reasoning applies if $J_{r} \in F_{n}$. Let $B_{\max }$ and $B_{\min }$ be the maximum and minimum output power levels in $\mathrm{dBm}$, respectively, of the Bluetooth device under consideration. For $J_{t} \in H_{i}$, one can express $J_{t}$ as

$$
J_{t}=J_{t}^{(\min )}+(i+\xi) \Delta
$$

in which $\xi \in[0,1)$. Since $B_{\min } \leq J_{t} \leq B_{\max }$, we have that $M=\left\lfloor-\xi+\left(B_{\max }-J_{t}^{(\min )}\right) / \Delta\right\rfloor$ and $M^{\prime}=\left\lfloor\xi-\left(B_{\min }-J_{t}^{(\min )}\right) / \Delta\right\rfloor$ where $\lfloor x\rfloor$ is the integer less than or equal to $x$. Let $p_{i, i^{\prime}}$ be the transition probability of state $i$ entering into state $i^{\prime}$. It follows that

$$
\begin{aligned}
p_{i, i+n} & =\operatorname{Pr}\left\{J_{r} \in G_{n} \mid J_{t} \in H_{i}\right\}, \quad 1 \leq n \leq M-i-1 \\
p_{i, i-n} & =\operatorname{Pr}\left\{J_{r} \in F_{n} \mid J_{t} \in H_{i}\right\}, \quad 1 \leq n \leq M^{\prime}+i-1 \\
p_{i, M} & =\operatorname{Pr}\left\{J_{r} \in \cup_{n=M-i}^{\infty} G_{n} \mid J_{t} \in H_{i}\right\}, \quad i \neq M \\
p_{i,-M^{\prime}} & =\operatorname{Pr}\left\{J_{r} \in \cup_{n=M^{\prime}+i}^{\infty} F_{n} \mid J_{t} \in H_{i}\right\}, \quad i \neq-M^{\prime} \\
p_{i, i} & =1-\sum_{i^{\prime}=-M^{\prime}, i^{\prime} \neq i}^{M} p_{i, i^{\prime}} .
\end{aligned}
$$

Substituting (1), (5) and (6) into (7a)-(7d) yields

$$
\begin{aligned}
p_{i, i+n}=Q & \left(\frac{\gamma \sigma_{\mathrm{dB}}}{2}-\frac{n+i+\xi}{\frac{\sigma_{\mathrm{dB}}}{\Delta}}\right)-Q\left(\frac{\gamma \sigma_{\mathrm{dB}}}{2}-\frac{n-1+i+\xi}{\frac{\sigma_{\mathrm{dB}}}{\Delta}}\right), \\
1 \leq n \leq M-i-1 & (8 \mathrm{a}) \\
p_{i, i-n}=Q & \left(\frac{\gamma \sigma_{\mathrm{dB}}}{2}+\frac{n-1+S-i-\xi}{\frac{\sigma_{\mathrm{dB}}}{\Delta}}\right) \\
& -Q\left(\frac{\gamma \sigma_{\mathrm{dB}}}{2}+\frac{n+S-i-\xi}{\frac{\sigma_{\mathrm{dB}}}{\Delta}}\right), \\
& 1 \leq n \leq M^{\prime}+i-1 \\
p_{i, M}=1 & -Q\left(\frac{\gamma \sigma_{\mathrm{dB}}}{2}-\frac{M-1+\xi}{\frac{\sigma_{\mathrm{dB}}}{\Delta}}\right), \quad i \neq M \\
p_{i,-M^{\prime}=} & Q\left(\frac{\gamma \sigma_{\mathrm{dB}}}{2}+\frac{M^{\prime}-1+S+\xi}{\frac{\sigma_{\mathrm{dB}}}{\Delta}}\right), \quad i \neq-M^{\prime}
\end{aligned}
$$

where $Q(x)$ is the tail probability of the standard normal distribution and $S=\left(J_{r}^{(\mathrm{UT})}-J_{r}^{(\mathrm{LT})}\right) / \Delta$. The transition matrix, $\mathbf{T}_{\xi}$, is a function of $\xi$ and is given by $\mathbf{T}_{\xi}=\left[p_{i, i^{\prime}}\right]_{i, i^{\prime}=-M^{\prime}, \ldots, M}$. Let $P_{i}(\xi)$, conditioned on $\xi$, be the stationary probability of the state $i$ and denote $\mathbf{P}_{\xi}=\left[P_{-M^{\prime}}(\xi), \ldots, P_{M}(\xi)\right]$ as the state-probability vector. Note that $\mathbf{P}_{\xi}$ characterizes the distribution of transmit power. It is known [7] that $\mathbf{P}_{\xi}$, if exists, is an eigenvector of $\mathbf{T}_{\xi}$ with the corresponding eigenvalue equal to 1 and with $\sum_{i=-M^{\prime}}^{M} P_{i}(\xi)=1$. It is possible that $\mathbf{T}_{\xi}$ has multiple unity eigenvalues. In such case the distribution is not unique. We find that this situation occurs when $\sigma_{\mathrm{dB}}$ is too low (less than $2 \mathrm{~dB}$ for a GRPR less than $26 \mathrm{~dB}$ ). This situation is not dealt with in this letter as it will be shown that the range of $\sigma_{\mathrm{dB}}$ of interest is 3-6 dB. Note that the uniqueness and existence of $\mathbf{P}_{\xi}$ implies that the distribution of transmit power is independent of the level of initial transmit power.

We model that $\xi$ is a uniform random variable over $[0,1)$. This assumption is made in order to compute a representative value of mean transmit power for different Bluetooth devices, which may have different initial transmit power and hence different $\xi$. The mean transmit power in $\mathrm{mW}$ is given by $\overline{W_{t}}=E\left\{10^{J_{t} / 10}\right\}$ wherein $J_{t}$ is given by (6) and the expectation is taken over all the states and the random variable $\xi$. Evaluating $\overline{W_{t}}$ and substituting the resultant expression into (4), we get

$$
A_{\mathrm{dB}}=10 \log _{10}\left(\int_{0}^{1} \sum_{i=-M^{\prime}}^{M} P_{i}(\xi) \times 10^{(i+\xi) \Delta / 10} d \xi\right) .
$$

The integral can be easily computed by a numerical technique such as Simpson's rule.

\section{NUMERICAL RESUlTS AND CONCLUSIONS}

Since our goal is to evaluate the impact of shadowing rather than that of $B_{\max }$ or $B_{\min }$ on the mean transmit power, we consider the situation that the effect of $B_{\max }$ and $B_{\min }$ on $\overline{W_{t}}$ can be ignored. In computing the numerical results, values of $B_{\max }$ and $B_{\min }$ were selected such that $P_{M}(\xi)$ and $P_{-M^{\prime}}(\xi)$ were negligible when compared to stationary probabilities of other states. For example, we found that $B_{\max }=J_{t}^{(\min )}+30 \mathrm{dBm}$ and $B_{\min }=J_{t}^{(\min )}-10 \mathrm{dBm}$ satisfied this condition when GRPR $=20 \mathrm{~dB}, \Delta=2 \mathrm{~dB}$ and $2 \mathrm{~dB} \leq \sigma_{\mathrm{dB}} \leq 8 \mathrm{~dB}$.

The accuracy of $A_{\mathrm{dB}}$ given by (9) is first verified by simulation. The conditions under investigation are: GRPR $=20 \mathrm{~dB}$; $\Delta=2 \mathrm{~dB}$; and $\sigma_{\mathrm{dB}}$ ranges from $2 \mathrm{~dB}$ to $8 \mathrm{~dB}$. In the simulation, $J_{r}$ was computed by (1) with $U$ randomly generated, followed by adjusting $J_{t}$ according to the power-control algorithm. This process was repeated for 20000 times if $\sigma_{\mathrm{dB}}=2 \mathrm{~dB}$ and 200 times for other cases of $\sigma_{\mathrm{dB}}$ and a final value of $J_{t}$ was reached. The need to repeat 20000 times when $\sigma_{\mathrm{dB}}=2 \mathrm{~dB}$ is a result of slow convergence of state probabilities. We generated a total of 100000 final values of $J_{t}$, based on initial $J_{t}$ values randomly generated over a range between $J_{t}^{(\mathrm{min})}-40 \mathrm{dBm}$ and $J_{t}^{(\min )}+60 \mathrm{dBm}$. Based on the final $J_{t}$ values, the mean transmit power was computed and an estimation of $A_{\mathrm{dB}}$ was obtained. Table I lists the simulated values of $A_{\mathrm{dB}}$ and those values computed by (9). It is apparent that (9) gives a value of $A_{\mathrm{dB}}$ that is in close agreement to the simulated one.

Table I also lists the values of $A_{\mathrm{dB}}$ computed by (9) for different combinations of $\sigma_{\mathrm{dB}}$ and $\Delta$. The GRPR is $20 \mathrm{~dB}$. It is apparent that for a given $\sigma_{\mathrm{dB}}$, the step size $\Delta=2 \mathrm{~dB}$ yields the lowest $A_{\mathrm{dB}}$ among the three choices of $\Delta$, though the advantage is minor. Hence, using a smaller step size yields a lower mean 
TABLE I

(a) COMPuTEd VALUes of $A_{\mathrm{dB}}$ AgAinst $\Delta$ AND $\sigma_{\mathrm{dB}}(\mathrm{GRPR}=20 \mathrm{~dB})$. (b) Simulation Results OF $A_{\mathrm{dB}}$ FOR $\Delta=2 \mathrm{~dB}$

\begin{tabular}{ccccc}
\hline & $\overbrace{4}$ & (a) & & (b) \\
$\sigma_{\mathrm{dB}}$ & $\Delta=2 \mathrm{~dB}$ & $\Delta=4 \mathrm{~dB}$ & $\Delta=8 \mathrm{~dB}$ & $\Delta=2 \mathrm{~dB}$ \\
\hline $2 \mathrm{~dB}$ & 10.5 & 10.6 & 11.1 & 10.6 \\
$3 \mathrm{~dB}$ & 11.1 & 11.2 & 11.7 & 11.3 \\
$4 \mathrm{~dB}$ & 12.0 & 12.2 & 12.8 & 12.1 \\
$5 \mathrm{~dB}$ & 13.4 & 13.6 & 14.2 & 13.4 \\
$6 \mathrm{~dB}$ & 15.0 & 15.3 & 16.2 & 15.1 \\
$7 \mathrm{~dB}$ & 17.1 & 17.5 & 18.6 & 17.3 \\
$8 \mathrm{~dB}$ & 19.2 & 20.2 & 21.5 & 19.8 \\
\hline
\end{tabular}

transmit power. We should mention that using a smaller step size results in slower convergence to the desired transmit power. Designers of Bluetooth systems should also take this factor into consideration in determining $\Delta$. In the following, we consider the case that $\Delta$ is equal to $2 \mathrm{~dB}$ in the comparison of $A_{\mathrm{dB}}$ for different $\sigma_{\mathrm{dB}}$. It is apparent that a higher $\sigma_{\mathrm{dB}}$ yields a higher $A_{\mathrm{dB}}$, implying that more transmit energy is consumed if the channel is subject to more severe shadowing. For in-building propagation, the typical value of $\sigma_{\mathrm{dB}}$ ranges from $3 \mathrm{~dB}$ to 6 $\mathrm{dB}$ [5]. Results of Table I show that the mean transmit power varies by $3.9 \mathrm{~dB}$ over this range of $\sigma_{\mathrm{dB}}$. It is also noticed that for $\sigma_{\mathrm{dB}}=3$ and $6 \mathrm{~dB}$, respectively, a Bluetooth device needs to consume 11.1 and $15.0 \mathrm{~dB}$ more in the transmit energy than the minimum one that achieves an acceptable performance in the absence of shadowing.

Fig. 1 plots $A_{\mathrm{dB}}$ against $\sigma_{\mathrm{dB}}$ for $\Delta=2 \mathrm{~dB}$ and GRPRs $=26$, 20 , and $14 \mathrm{~dB}$. Note that 14 and $26 \mathrm{~dB}$ are, respectively, the lower and upper limits of GRPR specified in the specification [1] and that $20 \mathrm{~dB}$ is the nominal value. It is apparent that an increase in GRPR increases $A_{\mathrm{dB}}$, indicating that more transmit energy is used. In addition, the results indicate that $\sim 3$-dB increase (decrease) in the required mean transmit power is obtained if the GRPR is increased (decreased) by $6 \mathrm{~dB}$ relative to the nominal value of $20 \mathrm{~dB}$. Thus, the transmit energy consump-

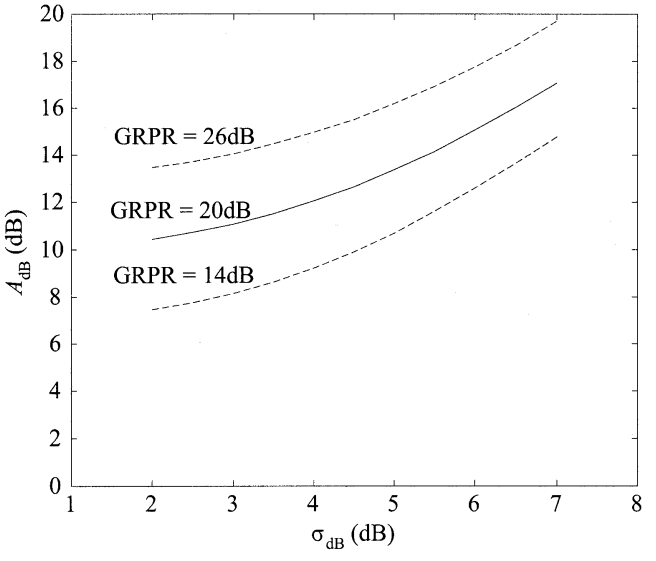

Fig. 1. $A_{\mathrm{dB}}$ against $\sigma_{\mathrm{dB}}$ for different GRPR's.

tion varies by around $6 \mathrm{~dB}$ among Bluetooth devices as a result of the $\pm 6-\mathrm{dB}$ tolerance in the GRPR.

\section{REFERENCES}

[1] "Specification of the Bluetooth System: Core," Bluetooth SIG, vol. 1.1, 2001.

[2] A. Jalali and P. Mermelstein, "Effects of diversity, power control and bandwidth on the capacity of microcellular CDMA systems," IEEE J. Select. Areas Commun., vol. 12, pp. 952-961, June 1994.

[3] M. L. Sim, E. Gunawan, B.-H. Soong, and C.-B. Soh, "Performance study of close-loop power control algorithms for a cellular CDMA system," IEEE Trans. Veh. Technol., vol. 48, pp. 911-921, May 1999.

[4] D. W. Paranchych, "On the performance of fast forward link power control in IS-2000 CDMA networks," in Proc. IEEE WCNC 2000, Sept. 23-28, 2000, pp. 603-607.

[5] G. Hess and M. Marsan, "Operational characteristic of transportable in-building propagation," in Proc. IEEE GLOBECOM'94, Dec. 2, 1994, pp. $37-41$.

[6] E. L. Crow and K. Shimizu, Lognormal Distributions: Theory and Applications, E. L. Crow and K. Shimizu, Eds. New York: Marcel Dekker, 1988, p. 9.

[7] A. Papoulis, Probability, Random Variables and Stochastic Processes, 3rd ed. New York: McGraw-Hill, 1991, p. 639. 